\title{
Percutaneous vertebroplasty for painful spinal metastasis: a good option for better quality of life
}

\author{
Young-Kwon Ko, and Yoon-Hee Kim \\ Department of Anesthesiology and Pain Medicine, Chungnam National University School of Medicine, Daejeon, Korea
}

Vertebral compression fracture (VCF) is one of the main causes of severe pain originating from the spine. Significant pain can lead to reduced physical activity and cause depression, deep vein thrombosis, pneumonia, and sores [1]. VCF is caused by osteoporosis, cancer (primary or metastatic cancer), or osteonecrosis. Treatments include bed rest, analgesics, back braces and other conservative treatments and more invasive procedures including percutaneous vertebroplasty (PVP) and open surgery [2].

In the recent guidelines of the Cardiovascular and Interventional Radiology Society, indications for PVP include: painful osteoporosis VCF refractory to medical treatment for the duration of more than 3 weeks, painful vertebrae due to aggressive primary bone tumor (hemangioma, giant cell tumor), painful vertebrae with extensive osteolysis due to malignant infiltration (multiple myeloma, lymphoma, metastatic cancer), painful fracture associated with osteonecrosis (Kummel's disease), reinforcement of the pedicle or vertebral body prior to posterior surgical stabilization procedures and chronic traumatic fracture in normal bone with nonunion of fracture fragments or internal cystic changes [2]. PVP has recently been implied in malignant vertebral compression fractures (MVCF) with positive results.

$90 \%$ of vertebral column tumors are caused by metastasis from other organs. The vertebral column is the most common site of metastasis within the skeletal system, with $70 \%$ of diagnosed patients showing bone metastasis [3]. Sites of metastasis break down to $60-80 \%$ in the thoracic spine, $15-$ $30 \%$ in the lumbar spine and $<10 \%$ in the cervical spine [4].
In $30 \%$ of vertebral metastasis from solid tumors, VCF occurs by reduction of vertebral bone strength via osteoblastic or osteoclastic activity [5]. MVCF can lead to economic loss, severe pain, neurologic injury, decreased life quality and even death.

In this edition of the Korean Journal of Anesthesiology, Seo et al. [6] reported the effectiveness of PVP at C7 for the treatment of painful metastasis. Patients with breast cancer metastasis at C7 afflicted with continuous severe weight-bearing neck pain despite radiation therapy underwent PVP via the anteromedial approach, which resulted in alleviated pain symptoms and increased physical activity. Therefore, PVP may be an option for the treatment of metastatic osteolytic vertebral lesions in the cervical spine for the purpose of alleviating intractable axial neck pain.

Early diagnosis and treatment of MVCF can preserve the patient's quality of life and the possibility of physical activities. Conventional treatments include surgery, chemotherapy, hormone therapy, and medical therapy. Although treatment can increase the median survival rate, for most patients, the purpose of treatment is reduction of pain, local disease progression, spinal instability, and neurologic complications. Pharmacotherapy using anti-inflammatory drugs (NSAID) and opioids are first used to treat pain but requires extended periods of bed rest. Since cancer and its treatment reduce the patient's immunity and cause the patient to be in a hyper-coagulation state, prolonged bed rest may increase morbidity from infection, pneumonia, deep vein thrombosis, and pulmonary embolism [7]. External beam radiation therapy (EBRT) is effective against pain but requires at least 2 weeks to obtain results. Also, since

Corresponding author: Yoon-Hee Kim, M.D., Ph.D., Department of Anesthesiology and Pain Medicine, Chungnam National University School of Medicine, Munhwa-ro, Jung-gu, Daejeon 301-721, Korea. Tel: 82-42-280-7841, Fax: 82-42-280-7968, E-mail: yhkim040404@gmail.com (c) This is an open-access article distributed under the terms of the Creative Commons Attribution Non-Commercial License (http:// creativecommons.org/licenses/by-nc/3.0/), which permits unrestricted non-commercial use, distribution, and reproduction in any medium, provided the original work is properly cited. 
continuous conventional EBRT can injure normal tissues and the nearby spinal cord, the procedure is limited by inevitable reduction of radiation dosage. Recent usage of stereotactic body radiotherapy (SBRT), a selective radiation therapy, showed positive effects as well as the spinal cord sparing effect, resulting in reduced pain in $84 \%$ of MVCF patients due to its higher radiation dosage and safety [8]. However, compared to PVP, complete alleviation of pain takes longer to manifest and usage of only SBRT cannot prevent spinal mechanical stability or bone compression fracture. Boehling et al. [9] suggested that SBRT is associated with a significant risk $(20 \%)$ of VCF. Risk factors for VCF include age $>55$ years, preexisting fracture, and existing pain. These risk factors may aid in the selection of which spinal SBRT patients should be considered for prophylactic vertebral stabilization or PVP. Open surgeries are performed for MVCF patients mainly to achieve decompression of the spinal cord and spinal stability, but can only be performed in rare cases because of the patient's short expected lifetime, medical comorbidities, long recovery time, and decreased life quality. PVP is a minimally invasive method that can be performed under local anesthesia with few complications; it can leading to rapid and effective pain reduction with mechanical stability [10].

Pain reduction after PVP is due to increased spine stability, tumor necrosis, and sensory nerve ending destruction. Tissue destruction occurs through highly exothermic reactions and local cytotoxic effects of polymethyl methacrylate (PMMA) polymerization [11]. Furthermore, PMMA not only acts as an analgesic but also has antitumor effects. PMMA applied to a malignant vertebral body can cause tumor necrosis through its cytotoxic activity, thermal effect, and ischemia effect. Spaceoccupying cement blocks tumor cell growth. Tumor feeding vascular structures are destroyed by heat, cytotoxic and chemical effects during PMMA polymerization, small nerve fiber injury and vascular obstruction due to compression after PMMA solidification. This leads to tumor ischemia and necrosis [12].

Patients who undergo PVP for VCF have rapid and dramatic reduction in pain within 24 hours. Gangi et al. [2] reported significant pain relief in $60-85 \%$ in MVCF and $80 \%$ in hemangioma, and showed a $91 \%$ reduction with the use of analgesics. Bone cement prevents further spinal fractures by providing stabilization and restoring vertebral body height. Significant pain reduction and spine stabilization significantly increase physical activity and improve quality of life. Less use of analgesics reduces complications such as nausea, vomiting, constipation and sedation, leading to increase in appetite. Although PVP is a minimally invasive technique with few contraindications, one must be aware of high complication risks in patient with osteomyelitis, discitis, active systemic infection, and un-corrective coagulopathy. PVP surgery can lead to results ranging from symptomless minor complications to major complications requiring surgical intervention such that they may result in significant disabilities or even death. Symptomatic complication rate for PVP in MVCP treatment is about 5\% but higher than osteoporotic VCF. Cement leakage is usually asymptomatic, with transient neurological deficits occurring in $1 \%$ of osteoporotic patients and in $5 \%$ of MVCP patients, with most problems disappearing within 30 days without the need for surgical intervention [2,13]. The reason behind the higher rate of complications in MVCF compared to that in osteoporotic VCF is due to the destruction of the vertebral body posterior cortex and the medioinferior cortex's pedicle by the tumor which leads to PMMA leakage into the spinal canal or the intervertebral foramen. To reduce PMMA leakage, if radiologic findings show destruction of the cortex, the amount of cement used must be limited and placed in the anterior vertebral body. PMMA leakage can occur in the paravertebral vein and inferior vena cava, thus, there is a $4-6.8 \%$ chance of asymptomatic pulmonary embolism [14]. Risks of symptomatic pulmonary embolism or paradoxical cerebral infarction due to medical and/or physical comorbidities are especially high in MVCF patients. To reduce such risks including cement leakage, injection of high viscosity cement in small volumes is recommended. Furthermore, reports of lower chances of risks in the treatment of MVCF by kyphoplasty instead of PVP are on the rise, resulting in more frequent usage of the treatment itself. Kyphoplasty inserts an inflatable bone tamp into the vertebral body inflation, creating a cavity in the inserted area. Since a larger volume of cement can be inserted using low pressure into the cavity this way, it is theoretically advantageous to achieve restoration of vertebral body height and reduction of cement leakage. However, Cloft and Jensen [15] claimed that kyphoplasty offers no significant advantage over vertebroplasty in terms of pain relief, vertebral body height restoration, and complication rate. Mathis [16] also reported that kyphoplasty cannot be said to be more evidence based or cost-effective than vertebroplasty.

PVP is effective for pain relief and spine stabilization concerning MVCP but has a limited antitumor effect. Recent reports of combined therapies with procedures of anticancer modality have shown promising results. Gerszten and Monaco [17] suggest that this treatment paradigm for pathological fracture of percutaneous transpedicular corpectomy combined with cement augmentation followed by radiosurgery was found to be safe and clinically effective. Hirsch et al. [18] suggested that longer-term palliation is best achieved using a multimodal approach of PVP and radiotherapy for MVCF. As such, PVP and EBRT are complementary procedures; PVP is effective for pain relief and spine stabilization while EBRT is effective for tumor treatment. Although there are few reports concerning 
the order of administration, Qian et al. [19] reported that first administrating PVP and then EBRT will allow continuous pain relief and spine stabilization and prevent further compression fracture. Furthermore, as EBRT causes vertebral body hardening while not affecting the efficacy of bone cement or its mechanical property, it will cause difficulties in administrating PVP. Therefore, he recommends first administrating PVP then EBRT. Also, in alternative radiation modalities, administering radioisotope during the PVP procedure has also been reported to be effective. Zuozhang et al. [20] reported that pain was relieved after PVP and I-125 isotope seed implantation for MVCF, and an MRI review two months after the surgery demonstrated a complete disappearance of the vertebral softtissue mass posterior to the vertebral body, and after two years of follow-up a functionally well-recovered spine with no signs of local recurrence was visible on MRI. Another method reported to be effective in spine stabilization is debulking of a tumor by percutaneous plasma-radiating radiofrequency ablation along with PVP [21].

In conclusion, a multidisciplinary team approach is the best way to accomplish cost-effective and fast treatment for MCVF and to improve the quality of life for the patients. In particular, efficiency of minimal invasive procedures such as PVP and EBRT has been shown in the recent rise of such treatments for MCVF.

\section{References}

1. Stallmeyer MJ, Zoarski GH, Obuchowski AM. Optimising patient selection in percutaneous vertebroplasty. J Vasc Interv Radiol 2003; 14: 683-96.

2. Gangi A, Sabharwal T, Irani FG, Buy X, Morales JP, Adam A. Quality assurance guidelines for percutaneous vertebroplasty. Cardiovasc Intervent Radiol 2006; 29: 173-8.

3. Aaron AD. The management of cancer metastatic to bone. JAMA 1994; 272: 1206-9.

4. Coleman RE. Metastatic bone disease: clinical features, pathophysiology and treatment strategies. Cancer Treat Rev 2001; 27: 165-76.

5. Bartels RH, van der Linden YM, van der Graaf WT. Spinal extradural metastasis: review of current treatment options. CA Cancer J Clin 2008; 58: 245-59.

6. Seo SS, Lee DH, Kim HJ, Yoon JW, Kwon OS, Kim KH. Percutaneous vertebroplasty at $\mathrm{C} 7$ for the treatment of painful metastases. Korean J Anesthesiol 2013; 64: 276-9.
7. Papaioannou A, Watts NB, Kendler DL, Yuen CK, Adachi JD, Ferko N. Diagnosis and management of vertebral fractures in elderly adults. Am J Med 2002; 113: 220-8.

8. Sahgal A, Ma L, Gibbs I, Gerszten PC, Ryu S, Soltys S, et al. Spinal cord tolerance for stereotactic body radiotherapy. Int J Radiat Oncol Biol Phys 2010; 77: 548-53.

9. Boehling NS, Grosshans DR, Allen PK, McAleer MF, Burton AW, Azeem S, et al. Vertebral compression fracture risk after stereotactic body radiotherapy for spinal metastases. J Neurosurg Spine 2012; 16: 379-86.

10. Tancioni F, Lorenzetti MA, Navarria P, Pessina F, Draghi R, Pedrazzoli $\mathrm{P}$, et al. Percutaneous vertebral augmentation in metastatic disease: state of the art. J Support Oncol 2011; 9: 4-10.

11. Galibert P, Deramond H. Percutaneous acrylic vertebroplasty as a treatment of vertebral angioma as well as painful and debilitating diseases. Chirurgie 1990; 116: 326-34.

12. Yang HL, Sun ZY, Wu GZ, Chen KW, Gu Y, Qian ZL. Do vertebroplasty and kyphoplasty have an antitumoral effect? Med Hypotheses 2011; 76: 145-6.

13. Deramond H, Depriester C, Galibert P, Le Gars D. Percutaneous vertebroplasty with olymethylmethacrylate. Technique, indications, and results. Radiol Clin North Am 1998; 36: 533-46.

14. Bernhard J, Heini PF, Villiger PM. Asymptomatic diffuse pulmonary embolism caused by acrylic cement: an unusual complication of percutaneous vertebroplasty. Ann Rheum Dis 2003; 62: 85-6.

15. Cloft HJ, Jensen ME. Kyphoplasty: an assessment of a new technology. AJNR Am J Neuroradiol 2007; 28: 200-3.

16. Mathis JM. Percutaneous vertebroplasty or kyphoplasty: which one do I choose? Skeletal Radiol 2006; 35: 629-31.

17. Gerszten PC, Monaco EA 3rd. Complete percutaneous treatment of vertebral body tumors causing spinal canal compromise using a transpedicular cavitation, cement augmentation, and radiosurgical technique. Neurosurg Focus 2009; 27; E9.

18. Hirsch AE, Jha RM, Yoo AJ, Saxena A, Ozonoff A, Growney MJ, et al. The use of vertebral augmentation and external beam radiation therapy in the multimodal management of malignant vertebral compression fractures. Pain Physician 2011: 14: 447-58.

19. Qian Z, Sun Z, Yang H, Gu Y, Chen K, Wu G. Kyphoplasty for the treatment of malignant vertebral compression fractures caused by metastases. Clin Neurosci 2011; 18: 763-7.

20. Zuozhang Y, Lin X, Hongpu S, Yunnchao H, Xiang M, Tao Y, et al. A patient with lung cancer metastatic to the fifth thoracic vertebra and spinal cord compression treated with percutaneous vertebroplasty and I-125 seed implantation. Diagn Interv Radiol 2011; 17: 384-7.

21. Georgy BA, Wong W. Plasma-mediated radiofrequency ablation assisted percutaneous cement injection for treating advanced malignant vertebral compression fractures. AJNR Am J Neuroradiol 2007; 28: 700-5. 